\title{
The Benefits of Curcuminoid to Expression Nuclear Factor Erythroid 2 Related Factor 2 (NRF2) and Signal to Noise Ratio (SNR) Value in the Noise Exposed Organ of Corti of Rattus Norvegicus
}

\author{
Tengku Siti Hajar Haryuna*1, Diana Amellya ${ }^{1}$, Delfitri Munir ${ }^{1}$, \\ Tengku Siti Harilza Zubaidah ${ }^{2}$
}

\begin{abstract}
Background: Noise-induced hearing loss (NIHL) can cause damage to the cochlea. Curcumin and nuclear factor erythroid 2-related factor 2 (NRF2) are transcription activators that play a crucial role in defence mechanisms against oxidative stress. The aim of this study was to determine the effect of curcuminoid administration on NRF2 expression, in the organ of Corti of cochlea of Rattus norvegicus that were exposed to noise, from the results of the distortion product otoacoustic emission (DPOAE) examination.

Methods: We divided 36 rats into six groups including Group 1 (control); Group 2 (noise exposure without curcuminoid administration); Group 3 (noise exposure+curcuminoid dose $100 \mathrm{mg} /$ day for four days); Group 4 (noise exposure+curcuminoid dose $200 \mathrm{mg} /$ day for four days); Group 5 (curcuminoid dose of $100 \mathrm{mg} /$ day for 14 days followed by two days of noise exposure); Group 6 (curcuminoid dose $200 \mathrm{mg} /$ day for 14 days followed by two days of noise exposure).

Results: Following noise exposure in rats, there was an effect/correlation between NRF2 expression, the SNR values obtained from DPOAE and curcuminoid administration.

Conclusions: There was a correlation between curcuminoid administration, NRF2 expression and DPOAE treatment. Following noise exposure in rats (Rattus norvegicus), SNR values obtained from DPOAE showed improved cochlear function.
\end{abstract}

Keywords: Curcuminoid, Distortion Product Otoacoustic Emission, Noise Induced Hearing Loss, Oxidative Stress, Nuclear factor erythroid 2-related factor 2.

\section{Introduction}

Hearing loss is a global problem (1). Noiseinduced hearing loss (NIHL) is the second most common cause of acquired hearing loss (2). According to the World Health Organization (WHO), hearing loss affects approximately 5$7 \%$ of the world's population (360-500 million people) (3).

NIHL is one of the most common forms of sensorineural hearing loss (4). One of the risk factors associated with NIHL is frequent exposure to noise levels greater than $85 \mathrm{~dB}$ for eight hours a day (5). Hearing loss caused by noise can be prevented by avoiding noise when surgery or medical treatment cannot be performed (6).

Over the last four decades, research has shown that otoacoustic emissions (OAEs) can be used to determine the differential diagnosis of 
sensory neural hearing loss, cochlear screening for infants, toddlers and other patients with hearing difficulties when exposed to loud noise, evaluation of external hair cells in patients with ototoxicity and other diseases (7).

OAEs are one of many diagnostic tests used in the field of audiology. The acoustic energy, which can be recorded through the ear canal, can be assessed using OAE (8). Distortion Product Otoacoustic Emission (DPOAE) has been recently used to detect NIHL and monitor the initial symptoms of cochlear dysfunction (9).

Curcumin is a yellow pigment in turmeric and curry spices that have broad pharmacological function (10). Curcumin is a species of Curcuma longa, a herbal monocot plant, that belongs to the ginger family (Zingiberaceae). It is prevalent in the tropical and subtropical regions of India, China, and Southeast Asia (11). Curcumin has antioxidative, anti-inflammatory, and chemotherapeutic potential (12).

Nuclear factor erythroid 2-related factor 2 (NRF2) is a major transcription factor that regulates antioxidant and detoxification genes, and plays an important role in defence mechanisms against oxidative stress due to its cytoprotective ability $(13,14)$. In contrast, curcumin can protect and activate NRF2 depending on the response in cellular or animal models during oxidative conditions (15). Curcumin also acts as an agonist to exogenous NRF2, can trigger nuclear translocation, and the biological effects of NRF2 itself (16).

In this study, we sought to explore whether there was a correlation between NRF2 expression and DPOAE treatment in the cochlea of rats exposed to noise following curcuminoid administration.

\section{Materials and Methods Design of study}

This study used a randomized post-test only control group laboratory experimental design. We acquired approval from the research ethics institute of the Faculty of Medicine, Universitas Sumatera Utara (No.509 / TGL / KEPK FK USU-RSUP HAM / 2018) before the start of the study.

\section{Procedures of animal care}

The study was conducted on 36 male Wistar strain rats (Rattus norvegicus). These rats were approximately 2-3 months old and weighed between 150 and 250 grams. The temperature and humidity were kept at $20-26{ }^{\circ} \mathrm{C}$, and 30 $70 \%$, respectively. They were provided adequate light, food, and water after gaining veterinarian clearance (17).

\section{The group of study}

Thirty-six rats were divided into six groups ( $\mathrm{n}=$ 6 ). The first group (Group 1) was the control group. Group 2 was subjected to noise $(110 \mathrm{~dB})$ for two hours during a two-day period, followed by two days of rest and no curcuminoid. Group 3 was given $100 \mathrm{mg}$ curcuminoid for four days and then exposed to noise $(110 \mathrm{~dB})$ for two hours over two days, followed by a two-day rest period. Group 4 was given $200 \mathrm{mg}$ curcuminoid for four days and exposed to noise $(110 \mathrm{~dB})$ for two hours over two days, followed by a two-day rest period. Group 5 received $100 \mathrm{mg}$ curcuminoid for 14 days and was subjected to noise $(110 \mathrm{~dB})$ for two hours across a two-day period, followed by a two-day rest period. Group 6 was given $200 \mathrm{mg}$ of curcuminoid for 14 days and subjected to $110 \mathrm{~dB}$ noise for two hours across a two-day period, followed by a two-day rest period.

\section{Procedure of noise exposure}

Noise treatment was conducted by placing rats on a box $(64.5 \times 45 \times 40 \mathrm{~cm})$ made of cork coated by foam with speakers attached to the roof and a hole at the base to measure intensity. Measurements were taken using a sound level meter at eight points in the cage where there was a noise difference not exceeding $1 \mathrm{~dB}$. The sound source was provided by a CD player, and an amplifier that produces noise at a frequency of $1-10 \mathrm{kHz}$ and intensity of $110 \mathrm{~dB}$ for two hours for two days.

\section{DPOAE examination}

DPOAE was performed three times using Elios Otodia (Echodia Ltd, London, UK); first before treatment, then two days after noise exposure, and finally two days following rest. The rats 
were initially anesthetized with ketamine at a dose of $90 \mathrm{mg} / \mathrm{kg} \mathrm{BW}$ and xylazine at a dose of $10 \mathrm{mg} / \mathrm{kg} \mathrm{BW}$, both of which were administered intraperitoneally $(18,19)$. The probe was made using a plastic micropipette that was adjusted to size and placed in the ear canal.

\section{Procedures of hematoxylin-eosin and immunohistochemical staining}

Following DPOAE, all rats were sacrificed, and a temporal bone necropsy was performed. The temporal bone was fixed with $10 \%$ formalin buffer solution followed by EDTA for four weeks until decalcification occurred. Next, each tissue sample was processed in a paraffin block and sliced into thick sections (4 $\mu \mathrm{M})$, which were then mounted on a slide and stained with hematoxylin-eosin and NRF2 H300: sc-13032 primary antibody (Santa Cruz, United States) for NRF2 expression. An Olympus $\mathrm{XC10}$ microscope was used to examine the expression of NRF2 at a magnification of $40 \mathrm{X}$. The distribution and intensity of these immunohistochemical (IHC) images were used to measure NRF2 expression. Distribution assessment was done by calculating the percentage of brown cells under the microscope. The Immunoreactive Score (IRS) formula was used to assess NRF2 expression by multiplying the percentage of brown cells with the intensity of the external appearance. The distribution of cells dyed brown was divided into four categories: $0=$ no cells dyed brown, $1=<10 \%$ cells dyed brown, $2=11-50 \%$ cells dyed brown, $3=51-80 \%$ cells dyed brown and $4=>81 \%$ cells dyed brown. Then, intensity was assessed by category: $0=$ unbroken, $1=$ weak intensity, $2=$ moderate intensity, $3=$ strong intensity. The calculated results showed a minimum score of 0 and a maximum score of 12 (20).

\section{Procedure of curcuminoid administration}

Curcuminoid extracted from Curcuma longa $\mathrm{L}$. (turmeric) was standardized to $16.62 \pm 0.14 \%$ w/w at the Faculty of Pharmacy Assessment Service Unit in Airlangga University. We were given curcuminoid in two doses (100 $\mathrm{mg}$ and $200 \mathrm{mg}$ ) in the form of a powder. Curcuminoid powder was suspended in $0.5 \%$ Carboxy Methyl Cellulose $(0.5 \mathrm{~g} \mathrm{CMC} \mathrm{in} 100 \mathrm{ml}$ of distilled water) before being administered directly to the stomachs of rats in a nasogastric tube. This solution was administered daily for four consecutive days in groups 3 and 4 and 14 consecutive days in groups 5 and 6 before noise exposure.

\section{Statistical analysis}

The data was analyzed using a One Way ANOVA test. To see the differences in each group, a Post Hoc Bonferroni test was performed. A Pearson Correlation test was used to find a correlation. Statistical tests were considered significant if the $\mathrm{p}$-value was less than $0.05(\mathrm{p}<0.05)$.

\section{Results}

We found differential expression in NRF2 in the organ of Corti of the cochlea (Figure 1). In group 2, we observed a decrease in NRF2 expression compared to controls. In groups 3, 4, 5 and 6, we observed an increase in NRF2 expression.

\section{Differences in the mean of SNR Value for each group.}

On days 0,2 , and 4 , there was no significant difference in the mean SNR values from DPOAE in group 1 ( $p>0.05$ ). However, there was a significant difference on day 0,2 , and 4 in groups $2,3,4,5$, and $6(\mathrm{p}<0.05)$ (Table 1$)$.

\section{Differences in NRF2 expression in the organ of corti of Rattus norvegicus cochlea in all groups}

In all groups, there was a significant difference in the average levels of NRF2 expression in the organ of Corti of the cochlea of Rattus norvegicus $(\mathrm{p}<0.05)$ (Table 2$)$.

\section{Correlation of SNR value NRF2 expression by curcuminoid administration.}

In all groups, there was a positive correlation between SNR values and NRF2 expression in the organ of Corti of Rattus norvegicus subjected to noise and curcuminoid injection $(\mathrm{p}<0.05)$ (Table 3). 

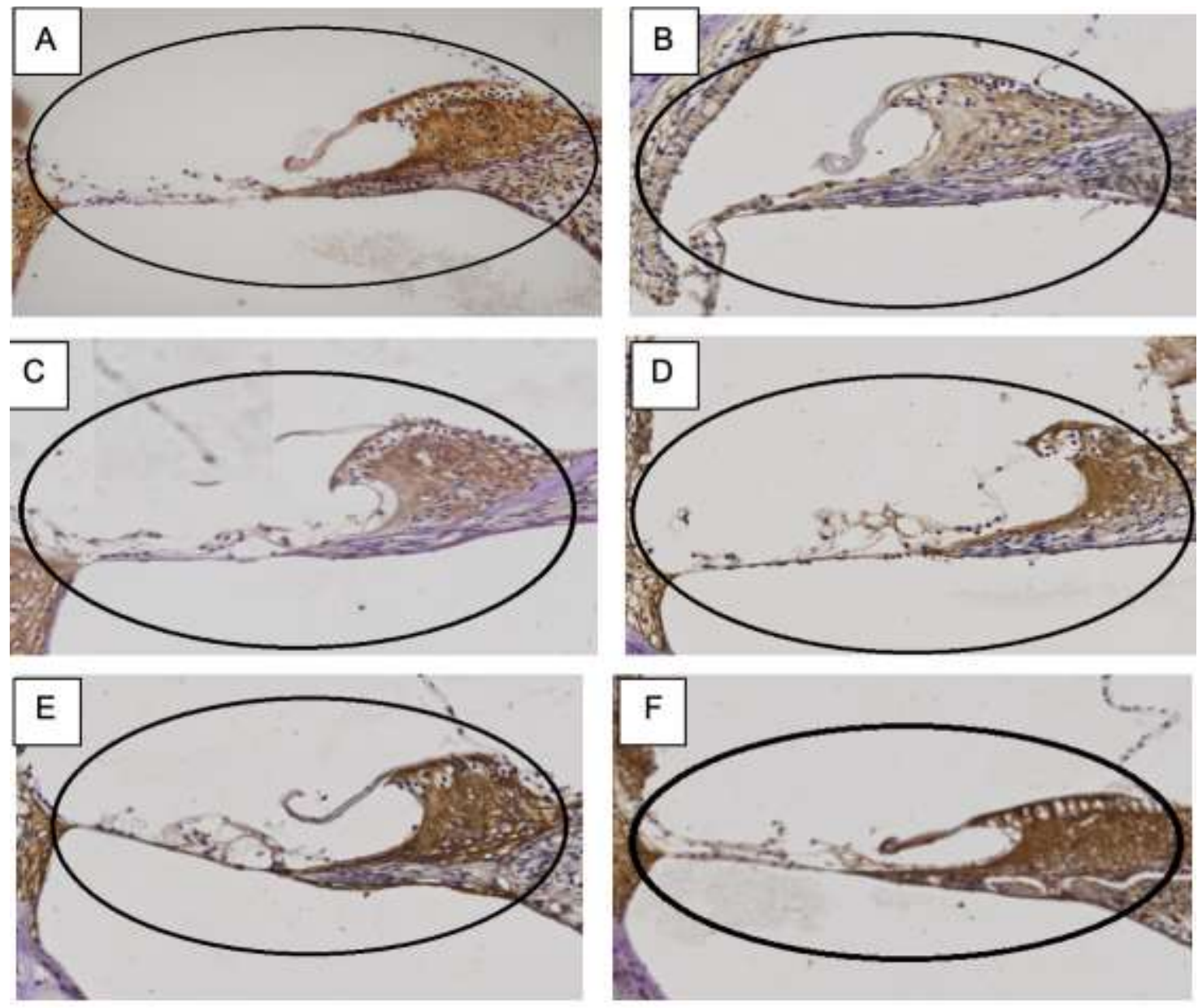

Fig. 1. An overview of NRF2 expression (at 40x magnification). The circle shows a picture of NRF2 expression in the organ of Corti of Rattus norvegicus cochlea marked in brown. A) group 1; B) group 2; C) group 3; D) group 4; E) group 5; F) group 6.

Table 1. Differences in the mean of SNR values following DPOAE on the Day 0,2 and 4 for each group.

\begin{tabular}{|c|c|c|c|c|}
\hline \multirow{2}{*}{$\begin{array}{l}\text { Group } \\
\text { SNR }\end{array}$} & \multicolumn{4}{|l|}{ Mean \pm SD } \\
\hline & Day 0 & Day 2 & Day 4 & p value \\
\hline Group 1, (control) & $11.62 \pm 3.11$ & $10.37 \pm 2.51$ & $9.30 \pm 2.11$ & 0.333 \\
\hline Group 2, (Noise $110 \mathrm{~dB})$ & $8.63 \pm 1.49$ & $4.13 \pm 0.98$ & $4.43 \pm 1.47$ & $<0.001$ \\
\hline Group 3, (Curative with curcuminoid 100 mg) & $9.57 \pm 1.71$ & $6.32 \pm 2.85$ & $8.41 \pm 1.08$ & 0.041 \\
\hline Group 4, (Curative with curcuminoid 200 mg) & $7.97 \pm 1.12$ & $4.52 \pm 1.37$ & $6.93 \pm 0.46$ & $<0.001$ \\
\hline Group 5, (Preventive with curcuminoid 100 mg) & $9.03 \pm 1.40$ & $5.45 \pm 0.52$ & $7.12 \pm 0.46$ & $<0.001$ \\
\hline Group 6, (Preventive with curcuminoid 100 mg) & $10.30 \pm 0.79$ & $5.20 \pm 0.25$ & $5.92 \pm 0.60$ & $<0.001$ \\
\hline
\end{tabular}


Table 2. Differences in NRF2 expression in the organ of Corti of the cochlea of Rattus norvegicus in all groups.

\begin{tabular}{llllc}
\hline \multicolumn{1}{c}{ Variable } & n & Mean \pm SD & Min-Max & p value \\
\hline Group 1, (Control) & 6 & $9.83 \pm 1.72$ & $8.00-12.00$ & $<0.001$ \\
\hline Group 2, (Noise 110 dB) & 6 & $2.32 \pm 0.95$ & $1.00-8.00$ & $<0.001$ \\
\hline Group 3 (Curative with curcuminoid 100 mg) & 6 & $2.66 \pm 1.08$ & $2.00-9.00$ & $<0.001$ \\
\hline Group 4 (Curative with curcuminoid 100 mg) & 6 & $1.97 \pm 0.80$ & $6.00-10.00$ & $<0.001$ \\
\hline Group 5, (Preventive with curcuminoid 100 mg) & 6 & $1.97 \pm 0.81$ & $6.00-12.00$ & $<0.001$ \\
\hline Group 6, (Preventive with curcuminoid 200 mg) & 6 & $1.67 \pm 0.68$ & $8.00-12.00$ & $<0.001$ \\
\hline
\end{tabular}

Table 3. Correlation between SNR values and NRF2 expression in the organ of Corti of the cochlea of Rattus norvegicus subjected to noise exposed and curcuminoid administration.

\begin{tabular}{lcc}
\hline Correlation & r (Pearson correlation) & p value \\
\hline SNR values with NRF2 expression in group II & $0.870^{*}$ & 0.024 \\
\hline SNR values with NRF2 expression in group III & $0.860^{*}$ & 0.028 \\
\hline SNR values with NRF2 expression in group IV & $0.827^{*}$ & 0.042 \\
\hline SNR values with NRF2 expression in group V & $0.829^{*}$ & 0.041 \\
\hline SNR values with NRF2 expression in group VI & $0.974^{*}$ & 0.001 \\
\hline
\end{tabular}

\section{Discussion}

Curcumin possesses anti-bacterial, anti-fungal, anti-viral, anti-inflammatory, anti-proliferative, and apoptotic properties $(21,22)$. Curcumin has been demonstrated in numerous studies to be hypoglycemic and of recent, curcumin has had therapeutic effects on cancer, diabetes, liver, cardiovascular, metabolic and autoimmune diseases $(23,24)$. Curcumin can modulate a wide range of molecular targets implicated in several stages of disease by regulating transcription factors, growth factors, receptors, cytokines, kinases, cell defence enzymes, metastases, and apoptotic molecules (22).

We conducted this study because there was little evidence in the literature on the effects of curcuminoid administration on NRF2 expression in the cochlea of Rattus norvegicus subjected to noise.

We found a significant difference in the average levels of NRF2 expression in the organ of Corti of Rattus norvegicus cochlea in all groups since NRF2 is a transcription factor and a redox-sensitive master regulator that has been associated with antioxidant stress and drug metabolism. NRF2 also protects normal cells from oxidative stress and electrophilic attack generated by harmful exogenous agents (25).

In this study, there was a change in NRF2 expression after curcuminoid administration. Yamaguchi conducted a study in 2017 using mice subjected to $90 \mathrm{~dB}$ of noise for one hour daily for five days. Following an Auditory Brainstem Response (ABR) check, they found a progressive shift in the hearing threshold. Then, they administered curcumin orally for three days daily before and during noise exposure. They found that this significantly reduced hearing loss. Therefore, curcumin can prevent hearing loss caused by repeated noise exposure suggesting that it has a preventative effect on NIHL and sensorineural hearing loss (25). This is similar to our work in which curcuminoids activated NRF2, as evidenced by the fact that after 
administering curcuminoids, NRF2 expression during IHC examination increased in groups 3, 4, 5 and 6.

Other studies suggest that curcumin administration can significantly protect cochlear morphology and function in paclitaxel-induced ototoxic mice using light microscopy and DPOAE to evaluate immunohistopathology, and functional changes in the auditory system. Curcumin is considered a dietary supplement derived from natural sources given to patients undergoing chemotherapy with paclitaxel (23).

Kopke demonstrated that by administering antioxidants prior to noise exposure can lower hearing thresholds and prevent cell death (26). In this study, we found that administering 100 $\mathrm{mg}$ or $200 \mathrm{mg}$ of curcumin for 14 days before noise exposure resulted in a significant difference in mean SNR values following DPOAE and NRF2 expression in all groups.

This study confirmed prior findings that NRF2 is a transcription activator that plays a crucial role in defence mechanisms against oxidative stress (14). Interestingly, the administration of curcuminoid can increase NRF2 expression which can help to treat NIHL patients. We showed that there was an effect/correlation between SNR values from DPOAE with NRF2 expression in the organ of

\section{References}

1. Zhou J, Shi Z, Zhou L, Hu Y, Zhang M. Occupational noise induced hearing loss in china: a systematic review and meta analysis. BMJ Open. 2020;10:e037596.

2. Loukzadeh Z, Hakimi A, Esmailidehaj M, Mehparvar AH. Effect of ascorbid acid on noised induced hearing loss in rats. Iranian Journal of Otorhinolaryngology. 2015;27(4):267-272.

3. Moos WH, Faller DV, Glavas IP, Harpp DN, Irwin $\mathrm{MH}$, Kanara I, Pinkert CA< Power WR, Steliou K, Vavvas DG, Kodukala K. A New Approach to Treating Neurodegenerative Otologic Disorders. Biores Open Access. 2018;7(1):107-115.

4. Le TN, Straatman LV, Lea J, Westerberg B. Current insights in noise-induced hearing loss: a
Corti of Rattus norvegicus cochlea that was exposed to noise. Finally, due to curcuminoid administration, there was an improvement in the cochlear function. As a result, this research could progress to clinical trials to prevent and treat the occurrence of NIHL in humans.

\section{Acknowledgements}

The author is very grateful to the Research Institute of the Universitas Sumatera Utara for the assistance of the TALENTA research fund and with the research implementation contract of the Universitas Sumatera Utara in the 2018 fiscal year with No: 2590/UN.5.1R/ PPM/2018 on March 16, 2018. The author is also grateful to the Department of Pathology Anatomy, Faculty of Medicine, Universitas Sumatera Utara. Department of Biochemistry, Faculty of Medicine, University of Brawijaya which has helped to provide scientific equipment.

This study was approved by the research ethics institute of the Faculty of Medicine, Universitas Sumatera Utara (No.509 / TGL / KEPK FK USU-RSUP HAM / 2018).

This study support by Research Institute of the Universitas Sumaera Utara for funding support under the TALENTA Research Implementation Contract of the Universitas Sumatera Utara 2019 fiscal year No. 2590 / UN.5.I.R /PPM / 2018 on March 16, 2018.

literature review of the underlying mechanism, pathophysiology, asymmetry, and management options. J Otolaryngol Head Neck Surg. 2017;46(1):41.

5. Kicher ED, Ocansey G, Abaidoo B, Atule A. Occupational hearing loss of the market mill workers in the city of Accra, Ghana. Noise Health. 2014;16(70):183-8.

6. World Health Organization. (2015). Hearing loss due to recreational exposure to loud sounds: a review. World Health Organization.

7. Martin LLB, Martin GK. Otoacoustic emissions disorders of peripheral and central auditory processing. Handbook of Clinical Neurophysiology. 2013.

8. Basner M, Babisch W, Davis A, Brink M, 
9. Clark C, Janssen, et al. Auditory and nonauditory effects of noise on health. Lancet. 2014;383(9925):1325-1332.

10. Meinke DK, Clavier OH, Norris J, Schoder RK, Allen L, Buckey JC. Distortion product otoacoustic product level maps from normal and damage cochlea. Noise Health. 2013;15(66):315-25.

11. Kao HH, Wu CJ, Won SJ, Shin JW, Liu HS, $\mathrm{Su}$ CL. Kinase gene expression and sunsellular protein expression patern of protein kinase c isoforms in curcumin treated human hepatocellular carcinoma hep 3B cells. Plant Foods Hum Nutr. 2011;66(2):136-42.

12. Trujillo J, Chirino YI, Jijon EM, AndericaRomero AC, Tapia E, Pedraza-Chaverri J. Renoprotective effect of the antioxidant curcumin: recent findings. Redox Bio. 2013; 17;1(1):448-56.

13. Hosseini S, Chamani J, Rahimi H, Azmoodeh $\mathrm{N}$, Ghasemi F, Abadi PH. An in vitro study on curcumin delivery by nano-michelles for esophageal squamous cell carcinoma (KYSE-30). Rep Biochem Mol Biol. 2018;6(2):137-143.

14. Mendez-garcia LA, Martinez-Castillo M, Villegas-Sepulveda N, Orozco L, Cordova EJ. Curcumin induces p53-independent inactivation of NRF2 during oxidative stress-induced apoptosis. Hum Exp Toxicol. 2019;38(8):951-961.

15. Honkura Y, Matsuo H, Murakami S, Sakiyama M, Mizutari K, Shiotani A, et al. NRF2 is a key target for prevention of noise-induced hearing loss by reducing oxidative damage of cochlea. Sci Rep. 2016;6:19329.

16. Reyes SG, Guzman-Beltran S, MedinaCampos ON, Pedraza-Chaverri J. Curcumin Pretreatment Induces Nrf2 and an Antioxidant Response and Prevents Hemin-Induced Toxicity in Primary Cultures of Cerebellar Granule Neurons of Rats. Oxid Med Cell Longev. 2013; 2013:801418.

17. Liu Z, Dou W, Zheng Y, Wen Q, Qin M, wang $X$, et al. Curcumin upregulated s NRF2 nuclear translocation and protects rat hepatic stellate cells against oxidative stress. Mol Med Rep. 2016;13(2):1717-24.

18. National Research Council (US) Committee for the Update of the Guide for the Care and Use of Laboratory Animals. Guide for the Care and Use of Laboratory Animals. 8th ed. Washington (DC): National Academies Press (US). 2011;8:41-90.

19. Albert Einstein College of Medicine Institute for Animal Studies. Recommended Methods of Anesthesia, Analgesia, and Euthanasia for Laboratory Animal Species. Part 1:296-308.

20. Toydemir T, Kanter M, Erboga M, Oguz S, Erenoglu C. Antioxidative, antiapoptotic and proliferative effect of curcumin on liver regeneration after partial hepatectomy in rats. Toxicol Ind Health. 2015;31(2):162-72.

21. Czogalla B, Kahaly M, Mayr D, Schmoeckel E, Niesler B, Kolben T, et al. Interaction of $\mathrm{ER} \alpha$ and NRF2 impacts survival in ovarium cancer patients. Int $\mathbf{J}$ Mol Sci. 2019;20(1):112.

22. Aggarwal B, Sung B. Pharmacological basis for the role of curcumin in chronic disease: an age-old spice with modern targets. Trends Pharmacol Sci. 2009;30(2):85-94.

23. Prasad S, Gupta SC, Tyagi AK, Aggarwal BB. Curcumin, a component. Of golden spice: from bedside to bench and back. Biotechnol Adv. 2014;32(6):1053-64.

24. Bucak A, Ozdemir C, Ulu S, Gonul Y, Aycicek A, Uysal M, et al. Investigation of protective role of curcumin against paclitaxelinduced inner ear damage in rats. Laryngoscope. 2015;125(5):1175-82.

25. Yamaguchi T, Yoneyama M, Onaka Y, Imaizumi A, Ogita K. Preventive effect of curcumin and its highly bioavailable preparation on hearing loss induced by single or repeated exposure to noise; a comparative and mechanistic study. J Pharmacol Sci. 2017;134(4):225-233.

26. Taheri Z, Aghdaei AH, Irani S, Modarressi MH, Noormohammadi Z. Evaluation of epigenetic demetylation of NRF2, a master transcription factor for antyoxidanr enzymes, in colorectal cancer. Rep Biochem Mol Biol. 2020;9(1):33-39.

27. Kopke RD, Jackson RL, Coleman JK, Liu J, Bielefeld EC, Balough BJ. NAC for nois`e: From the bench top to the clinic. Hear Res. 2007;226(12):114-25. 